\title{
Sostenibilidad ambiental del sistema de producción de café orgánico en la región Junín
}

\author{
Environmental sustainability of organic coffee production system in Junin
} Fernando Suca Apaza', Guido Suca Apaza², Raúl Siche Jara
Universidad Continental

\section{RESUMEN}

Objetivo: Evaluar la sostenibilidad ambiental del sistema productivo del café orgánico en la región Junín, tomando como unidad de análisis de estudio la provincia de Satipo. Métodos: Se utilizó el análisis de la emergía, ésta caracteriza y construye el sistema productivo desde un enfoque sistémico; se cuantifica y analiza los principales flujos de recursos naturales y económicos, luego se calcula e interpreta los indicadores emergéticos del sistema. Resultados: El aporte de la economía presenta un $43,99 \%$. Sobre los índices emergéticos, se obtuvieron una transformidad de $6,79 \mathrm{E}+05 \mathrm{seJ} / \mathrm{J}$, renovabilidad de $34,28 \%$, tasa de eficiencia emergética de 2,27 ; tasa de carga ambiental de 1,92; tasa de inversión emergética de 0,79 y un índice de sostenibilidad emergética de 1,19. Conclusiones: El sistema productivo de café orgánico de la región Junín presenta un buen nivel de organización; también presenta un mayor aporte de recursos de la naturaleza con respecto al aporte de la economía. Los indicadores emergéticos demostraron que el sistema de café orgánico presenta mayor eficiencia, mayor renovabilidad, menor impacto al ecosistema y buena contrapartida de la naturaleza a las inversiones de los productores. Por lo tanto, el sistema de producción de café orgánico de la región de Junín es sostenible ambientalmente.

1 Docente de la Universidad Nacional del Centro del Perú, investigador de la Universidad Continental

2 Investigador de la Universidad Continental.

3 Docente de la Facultad de Ciencias Agrarias de la Universidad Nacional de Truillo. 


\section{ABSTRACT}

Objectives: Evaluate the environmental sustainability of the production system of organic coffee at Junin region, taking as analysis unit the province of Satipo. Methods: it was used the emergy analysis, that characterizes and builds up the production system considering a systemic approach; it quantifies and analyzes the main fluxes of natural and economical resources; and then calculates emergy indicators. Results: The accounting of economy to the system is $43.99 \%$. About the emergy indexes, it was obtained a transformity value of $6.79 \mathrm{E}+05$ $\mathrm{seJ} / \mathrm{J}$; renewability percentage of $34.28 \%$; emergy yield ratio of 2.27 ; environmental loading ratio of 1.92; emergy investment ratio of 0.79 ; and the emergy sustainability index of 1.19. Conclusions: the production system of organic coffee at Junin Region presents a good organizational level; also shows a higher account of natural resources than the account of economy. The emergy indexes showed that the system of organic coffee presents the highest efficiency level, more renewability, less impact to ecosystem and better counterpart of the nature to the investment than the producers do. In conclusion, the production system of organic coffee at Junin Region is environmentally sustainable.

Keywords: environmental sustainability, emergy, organic coffee.

\section{INTRODUCCIÓN}

Esta investigación nace de la importancia de conocer el grado de sostenibilidad ambiental en el sistema de producción del café orgánico impulsado en la selva central del Perú, debido a que actualmente toda actividad desarrollada por el hombre debe ser compatibilizada con la ausencia de impactos negativos al medio ambiente.

En la actualidad, la producción de café orgánico en el país viene siendo desarrollada sobre la base de decisiones ausentes de análisis sostenible, debido probablemente a que el modelo de desarrollo sostenible es un tema muy reciente, y por lo tanto poco interiorizado, en los diferentes ámbitos de decisión de gobierno. Esta carencia trae, desde luego, consecuencias limitantes en el crecimiento y desarrollo del sistema productivo del café, las mismas que retrasan la aproximación hacia un modelo de crecimiento y desarrollo sostenible.

Los intentos de lograr una mayor sostenibilidad se manifiesta hoypromoviendo sistemas relacionados con el desarrollo sostenible como la implementación de sistemas de gestión ambiental; un mayor impulso del etiquetado de productos orgánicos y desarrollo de mercados para tales productos; un mayor fomento para generar un comercio más justo y que los productos sean comercializados en nichos de mercado específicos; y el impulso a las cadenas agroproductivas, entre otras acciones.

Esta situación exige la búsqueda de una solución al problema basada en metodologías científicas ya existentes como la emergía, que permitan analizar la sostenibilidad de todo el sistema, lo que en el Perú aún no se viene planteando.

Uno de los primeros trabajos científicos en el diagnóstico de la sostenibilidad fue en el 2007, en el que Siche (2) comparó y analizó los principales indicadores de sostenibilidad aplicados al caso pervano sobre la base de datos del 2004; entre las metodologías utilizadas estuvo la emergía. La aplicación de estos métodos mostró que la tendencia de la economía del Perú es de disminuir su sostenibilidad, que es explicada por la dependencia creciente de recursos no renovables, principalmente combustibles fósiles. Menciona que la sostenibilidad es buena (hasta el 2007) y que una disminución de su capacidad de soporte, como está ocurriendo, revertiría dicha condición.

Lomas, Di Donato y Ulgiati (3) manifiestan que la síntesis emergética es una 
contribución importante que permite tener una visión con un fuerte componente científico, llegando a producir, a partir del concepto de emergía, indicadores que permiten entender bajo un mismo marco de estudio los flujos de materia, energía, información y dinero ligados a sistemas agrícolas o tratar aspectos referentes al desarrollo, al comercio, entre otros. Esta referencia permite conocer la importancia de adoptar el método emergético como una herramienta fundamental en el análisis de la sostenibilidad del sistema agrícola del café.

Guillén (4) también utilizó el análisis emergético para determinar la sostenibilidad de sistemas agrosilvícolas y agroindustriales en México, donde la producción de café es considerada como un sistema agrosilvícola sostenible porque mantiene parte de la selva original.

La región Junín es una de las principales zonas cafetaleras del país. Las provincias productoras de café en la región Junín son actualmente Satipo y Chanchamayo. El interés que despierta el sistema productivo del café orgánico en Satipo, en especial, ha motivado para que esta provincia sea considerada como unidad de análisis en nuestra investigación.

Satipo está ubicado a 386 kilómetros de la ciudad de Lima y se caracteriza por su clima tropical, con pisos ecológicos de 400 a $1800 \mathrm{msnm}$. La principal actividad en la provincia es la agricultura y el cultivo más importante es el café, que representa el $50 \%$ del total del área agrícola.

En relación con su economía, se estima que el $50 \%$ de las familias son productoras de café, por lo que dependen fundamentalmente de este producto. La provincia de Satipo tiene ocho distritos, de los cuales San Martín de Pangoa alcanza la mayor producción de café con $43,8 \%$, seguida por Rio Negro con 32,5\% y Satipo con $17 \%$. La actividad cafetalera en esta provincia, según estimaciones económicas, genera 2960000 jornales al año. El valor del jornal de este producto oscila entre
15 y 20 soles, y la demanda se concentra principalmente en el período de cosecha.

Los productores están integrados en 57 organizaciones. Las formas más comunes de organización son asociaciones, cooperativas, central de asociaciones, comités y empresas comunales. Aproximadamente la mitad de estas agrupaciones están organizadas bajo la forma de asociación.

Los productores organizados representan el $28 \%$ del total del sector que conforman estos agentes productivos, en tanto que los no organizados, el $72 \%$. En la provincia existen pequeños y medianos productores que según su grado de organicidad podrían clasificarse en tres grandes grupos: productores no organizados, productores organizados con baja capacidad de gestión y productores organizados con mediana capacidad de gestión.

Las etapas identificadas del sistema de producción de café orgánico en Satipo son: producción, beneficio húmedo, transporte, poscosecha, control de calidad $y$ almacenamiento.

En la etapa de producción se realizan las siguientes labores culturales: poda, selección de brotes (deschuponado), fertilización, transporte de fertilizantes, manejo de sombra, deshierbo, control de plagas y cosecha del café cerezo. En la producción misma se utilizan herramientas, equipos y materiales.

El beneficio húmedo tiene la finalidad de seleccionar los granos de café cerezo para luego ser despulpados y posteriormente obtener el pergamino de café. Durante el proceso de beneficio húmedo se considera al agua como flujo renovable, al combustible como flujo no renovable y dentro de los materiales se encuentran la despulpadora, llaves, tuberías y materiales de construcción para las instalaciones de la planta de beneficio. Dentro de los servicios está considerada la mano de obra de los mismos productores. 
Los productores de café orgánico cuentan con infraestructura en las propias zonas donde están sus cultivos. Esta infraestructura forma parte de sus predios. Están divididas en las siguientes zonas: cuarto de herramientas, almacén de café, zona de beneficio húmedo, zona de fermentado y lavado, zona de aguas miel y compostera. El café pergamino húmedo o seco es transportado hacia los tendales o almacén de la cooperativa, es un servicio brindado a los socios productores. El costo del traslado es asumido por cada productor y depende de la distancia que se dé entre el predio del productor y la infraestructura de la cooperativa.

En las instalaciones de la cooperativa de Satipo se acopia el café de los socios productores. La liquidación de compra está en función de la humedad (12\%) y rendimiento (75\%). En cambio, los socios que desean hacer secar el café hasta alcanzar una humedad de $12 \%$ hacen uso del tendal de secado y guardiolas de la cooperativa. La planta de secado está ubicada en Río Negro, a $5 \mathrm{~km}$ del almacén de Satipo. Solo se considera el gasto por el servicio de secado en las guardiolas.

El objetivo de este estudio fue evaluar la condición de sostenibilidad ambiental del sistema de producción de café orgánico de la región Junín. La hipótesis fue que este sistema es sostenible ambientalmente utilizando el análisis emergético.

\section{MATERIAL Y MÉTODOS}

El alcance y diseño de este estudio fue descriptivo, transversal, desarrollado con datos del 2011 sobre el sistema de producción de café orgánico de la provincia de Satipo. Otros datos fueron recolectados a partir de los comités de café orgánico de Cana Edén, Vista Alegre, Río Venado, Huahuari, Satipo y Villa Victoria, los cuales pertenecen a la Cooperativa Agraria Cafetalera de Satipo. Se tomaron datos cualitativos y descriptivos por medio de entrevistas directas a los técnicos de la referida cooperativa y la revisión de documentos de las cooperativas de Satipo y Pangoa. Fue considerado un total de 12075 hectáreas de café orgánico. Esta información primaria y secundaria fue clasificada en los siguientes flujos: recursos renovables $(R)$, recursos no renovables $(N)$, materiales suministrados por la economía (M) y servicios suministrados por la economía (S).

La metodología utilizada fue la emergía desarrollada por Odum, basada en cuatro etapas: (a) la caracterización y elaboración del diagrama del sistema; (b) el análisis de los flujos identificados; (c) la obtención de los índices emergéticos y (d) la interpretación de los índices emergéticos (1).

En el diagrama del sistema, los flujos y las etapas productivas fueron organizados de izquierda a derecha, de acuerdo con la secuencia del proceso y de su transformidad. Así los elementos de la izquierda presentan menor emergía incorporada que los de la derecha. El límite del sistema fue claramente identificado. Luego se elaboró la tabla de flujos de emergía, donde cada flujo se convirtió en una línea de cálculo en la tabla de evaluación emergética (5). En esta tabla se consignó todas las contribuciones al sistema estudiado. Estas contribuciones se clasificaron en recursos renovables de la naturaleza, recursos no renovables de la naturaleza, materiales de la economía, servicios renovables de la economía y servicios no renovables de la economía. Posteriormente se cuantificaron cada uno de las 18 contribuciones. Se consideraron las siguientes unidades consistentes de flujos emergéticos: J/ha.año, kg/ha.año y S/./ha.año. En la siguiente columna se recopiló de fuentes secundarias los valores de transformidad por unidad compatible respecto a los flujos emergéticos. Finalmente se multiplicó el valor de los flujos de entrada y su respectiva transformidad de los 18 flujos emergéticos. Las unidades se expresaron en seJ/J, seJ/kg y seJ/S/. 


\section{RESULTADOS}

Como resultado de la primera etapa del análisis emergético, en la Figura 1 presentamos el diagrama sistémico completo, en la cual hemos delimitado las cuatro etapas principales del sistema, también denominados subsistemas: producción, beneficio húmedo, transporte y poscosecha. Paralelamente definimos el límite de todo el sistema productivo de café orgánico, en el que identificamos cada uno de los flujos que ingresan a los subsistemas. A cada uno de los subsistemas ingresa uno o más flujos de energía y está en función de la necesidad energética de cada subsistema.

De acuerdo con la metodología emergética, de las cuatro etapas mostradas en la Figura 1 se ha considerado tres subsistemas: producción, beneficio húmedo y postcosecha. El transporte es considerado una interacción debido a que interactúa entre dos subsistemas. También se puede ver en esta figura los elementos importantes para el funcionamiento del sistema de producción de café orgánico en la provincia de Satipo, los flujos simples o de menor intensidad a la izquierda, y los mayores y más complejos a la derecha. En este sistema la energía disponible es transformada en energías diferentes, de cantidades menores, que son aprovechadas en las etapas siguientes del sistema.

En la segunda etapa de la metodología emergética se cuantificó los principales recursos naturales y económicos que han sido incorporados en el sistema de producción de café orgánico. A continuación se describe cómo fueron calculados los 18 flujos emergéticos para el sistema de café orgánico:

Los flujos renovables como el sol indica la cantidad de energía (en Joule) que es igual al producto de la insolación media por el coeficiente de albedo. Éste es estimado por la diferencia del porcentaje total de radiación, equivalente a la unidad menos el porcentaje reflejado. El potencial químico de la lluvia fue calculada también en Joule. Es equivalente al producto de la precipitación, evapotranspiración, energía de la lluvia y densidad de la lluvia. De la misma manera, el agua de manantial es la energía del agua (en Joule) que es captada por reservorios

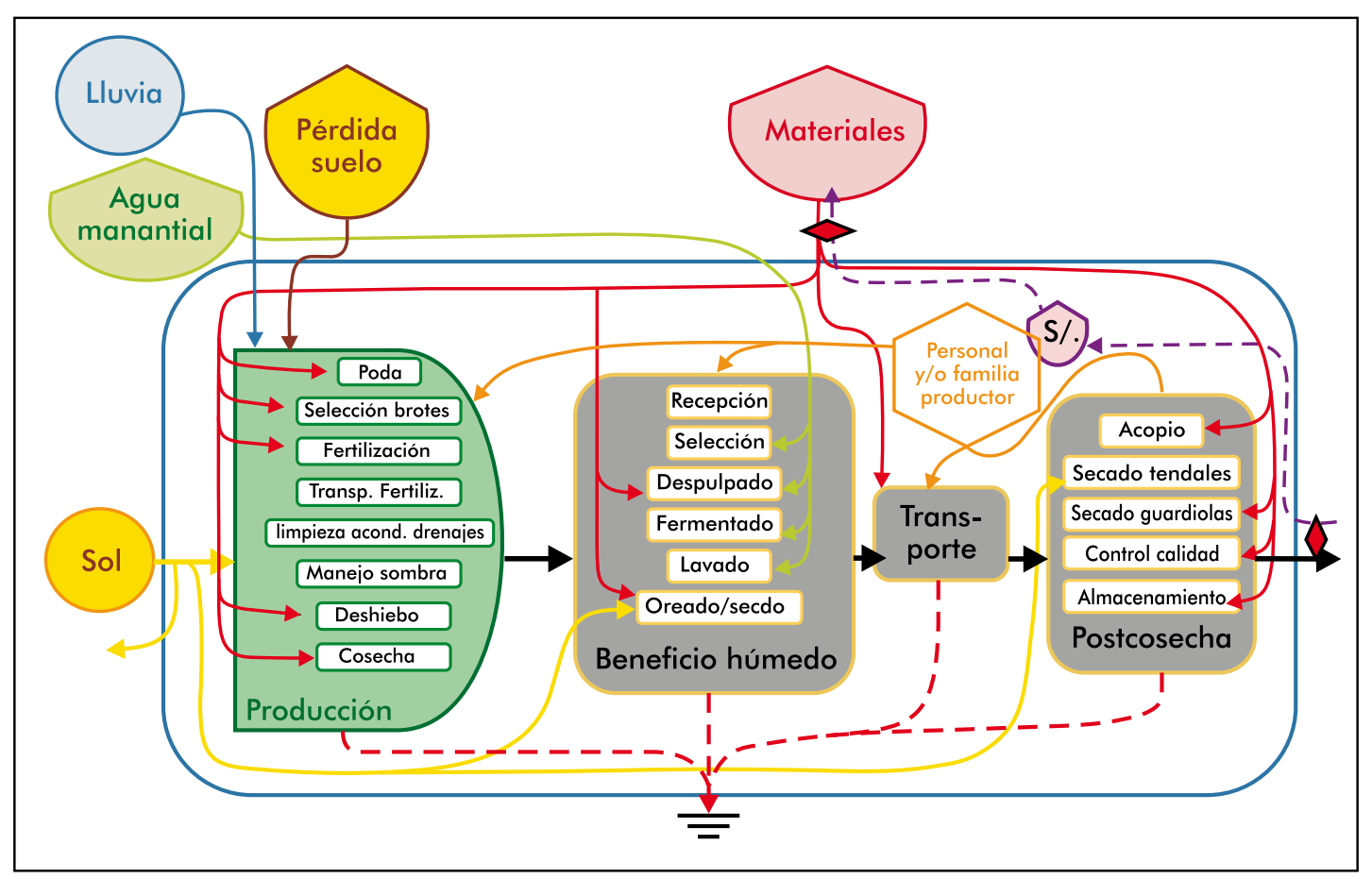

Figura Nº 1: Diagrama sistémico de la producción de café orgánico. 
naturales y luego transportada por tuberías de plástico hacia la planta de beneficio húmedo ubicado en el predio del productor.

Sobre los flujos no renovables, se ha considerado la pérdida de suelo. Este flujo es la pérdida selectiva de materiales del suelo ya sea por la acción del agua o viento. Se ha calculado en Joule y es el producto de la cantidad de pérdida de suelo, porcentaje de materia orgánica del suelo y la energía de la materia orgánica.

Los materiales de la economía, considerados no renovables de la economía, fueron calculados, para el caso de los abonos, como nitrógeno, fósforo y potasio absorbidos. Estos tres componentes se cuantificaron en $\mathrm{kg}$. Cada uno de ellos indican la diferencia de la cantidad de nutrientes que sale del sistema menos la cantidad que ingresa al sistema de producción de café; es decir, la cantidad de nutrientes que se analizó en muestras de café cerezo menos la cantidad de nitrógeno que se incorporó durante la fertilización con guano de isla y compost. El flujo de otros insumos corresponde al equivalente en nuevos soles (S/.). Estos insumos son en menor cantidad respecto a los abonos.

El flujo de la cantidad de combustible se ha calculado en Joule y representa la cantidad de flujo de energía producida por el total de combustible utilizado durante el despulpado del café. Se ha obtenido como producto del consumo de combustible, densidad del combustible, valor calórico del combustible, todo esto dividido entre el área total del subsistema de producción (12 075 ha).

El flujo de materiales y equipos se refiere a los materiales y equipos metálicos utilizados durante las etapas de todo el sistema. Este flujo es la razón del total de materiales y equipos entre el área total del café. El flujo de la infraestructura agrícola corresponde al monto de inversión de toda la infraestructura agrícola necesaria para todo el sistema. Fue calculado dividiendo el costo total de la inversión entre el promedio de vida útil de la infraestructura y entre el área total.
Los flujos del transporte son considerados no renovables de la economía. Dentro de estos flujos se ha calculado el de los vehículos, el cual es la cantidad (en kg) de los vehículos de transporte utilizados para trasladar el café y los insumos para el sistema. El cálculo fue a partir de la masa de los vehículos, producción total y rendimiento del café pergamino. El flujo de los neumáticos indica la cantidad (en kg) de los neumáticos de todos los vehículos para transportar el café y materiales del sistema. Este cálculo fue a partir del peso de los neumáticos, producción total y rendimiento del café. El flujo de combustible (en Joule) es la cantidad de flujo de energía producida por el total de combustible del transporte. Tal flujo se ha calculado como el producto del consumo, la densidad y el valor calórico del combustible.

Los siguientes flujos son considerados como no renovables de la economía y pertenecen al subsistema de poscosecha. El flujo de equipos poscosecha (en $\mathrm{kg}$ ) es la cantidad de masa de los equipos metálicos utilizados. Se ha calculado con los datos de masa, vida útil promedio de los equipos, producción y rendimiento del café pergamino. El flujo de energía eléctrica (en Joule) indica la cantidad de energía eléctrica consumida en las instalaciones de poscosecha del café entre la producción de café pergamino final. El flujo de infraestructura de la planta de secado (en S/.) es la inversión respecto a la vida útil de la infraestructura de la planta de secado y la producción de café durante la temporada 2011.

Dentro de los servicios se ha cuantificado el flujo de mano de obra (en Joule). Este flujo es la cantidad de energía aportada por el ser humano en las diferentes labores culturales del sistema de producción de café orgánico. Fue calculado en función de la cantidad de personal, consumo energético por día de un trabajador, número de días trabajados en el 2011 y el área de café del sistema. El referido flujo se ha desagregado en servicios renovables $y$ no renovables de la economía. El servicio considerado renovable significa $38 \%$ del total de energía de la mano de obra, y el 
no renovable, $62 \%$.

Luego fueron recopilados los valores de transformidad de fuentes secundarias, como lo establecen Fernandez (5) y Odum (1). Estas transformidades están expresadas en unidades compatibles respecto a los flujos emergéticos. Estos flujos se multiplicaron por sus respectivas transformidades, las cuales están expresadas en seJ/J, seJ/kg y seJ/S/. Finalmente, se obtuvo la emergía solar equivalente, de los 18 flujos emergéticos, en una sola unidad, el seJ/ha.año. En la Tabla 1 se aprecia los flujos identificados, sus transformidades y la cuantificación en una sola unidad.

En la Tabla 2 se presenta un resumen de los distintos grupos de flujos identificados. Dentro de los recursos renovables de la naturaleza se ha considerado los flujos de sol, lluvia y los servicios renovables de la economía. El flujo de agua de manantial ya no se ha considerado debido a un tema de

Tabla № 1: Flujos emergéticos del sistema de café orgánico

\begin{tabular}{|c|c|c|c|c|c|c|}
\hline Flujos & $\% R$ & Dato & Unid. & $\begin{array}{c}\text { Transformidad } \\
\text { (seJ/unid.) }\end{array}$ & Unidad & $\begin{array}{l}\text { Emergía solar } \\
\text { (seJ/ha.a) Total }\end{array}$ \\
\hline Renovables & & & & & & $2,17 E+15$ \\
\hline 1. Sol & 100 & $4,39 E+13$ & J/ha.año & 1 & $\mathrm{seJ} / \mathrm{J}$ & $4,39 E+13$ \\
\hline 2. Lluvia & 100 & $6,97 E+10$ & J/ha.año & $3,05 E+04$ & $\mathrm{seJ} / \mathrm{J}$ & $2,13 E+15$ \\
\hline \multicolumn{7}{|l|}{ Beneficio húmedo } \\
\hline 3. Agua manantial & 100 & $1,12 E+07$ & J/ha.año & $3,05 E+04$ & $\mathrm{seJ} / \mathrm{J}$ & $3,42 E+11$ \\
\hline No renovables & & & & & & $1,49 E+15$ \\
\hline 4. Pérdida de suelo & 0 & $1,20 E+10$ & J/ha.año & $1,24 \mathrm{E}+05$ & & $1,49 E+15$ \\
\hline Materiales & & & & & & $5,03 E+14$ \\
\hline 5. Nitrógeno & 0 & $2,42 \mathrm{E}+01$ & kg/ha.año & $6,38 E+12$ & $\mathrm{seJ} / \mathrm{kg}$ & $1,55 \mathrm{E}+14$ \\
\hline 6. Fósforo & 0 & $-2,72 \mathrm{E}+00$ & kg/ha.año & $6,55 E+12$ & $\mathrm{seJ} / \mathrm{kg}$ & $-1,78 E+13$ \\
\hline 7. Potasio & 0 & $2,49 \mathrm{E}+01$ & kg/ha.año & $2,92 \mathrm{E}+12$ & $\mathrm{seJ} / \mathrm{kg}$ & $7,26 E+13$ \\
\hline 8. Otros insumos & 0 & $2,59 E+01$ & S/./ha.año & $1,35 E+12$ & $\mathrm{seJ} / \mathrm{sol}$ & $3,49 E+13$ \\
\hline $\begin{array}{l}\text { 9. Combustible } \\
\text { (despulpado) }\end{array}$ & 0 & $2,15 E+08$ & J/ha.año & $5,50 E+04$ & $\mathrm{seJ} / \mathrm{J}$ & $1,18 E+13$ \\
\hline $\begin{array}{l}\text { 10. Materiales y } \\
\text { equipos (fierro) }\end{array}$ & 0 & $1,03 E+01$ & kg/ha.año & $1,13 E+13$ & $\mathrm{seJ} / \mathrm{kg}$ & $1,16 E+14$ \\
\hline $\begin{array}{l}\text { 11. Infraestructura } \\
\text { agrícola }\end{array}$ & 0 & $9,73 E+01$ & S/. /ha.año & $1,35 E+12$ & $\mathrm{seJ} / \mathrm{sol}$ & $1,31 E+14$ \\
\hline Transporte & & & & & & $7,45 \mathrm{E}+14$ \\
\hline 12. Vehículos & 0 & $6,34 \mathrm{E}+01$ & kg/ha.año & $1,13 E+13$ & $\mathrm{seJ} / \mathrm{kg}$ & $7,16 E+14$ \\
\hline 13. Neumáticos & 0 & $4,97 \mathrm{E}+00$ & kg/ha.año & $4,30 E+12$ & $\mathrm{seJ} / \mathrm{kg}$ & $2,14 \mathrm{E}+13$ \\
\hline 14. Combustible & 0 & $1,42 \mathrm{E}+08$ & J/ha.año & $5,50 \mathrm{E}+04$ & $\mathrm{seJ} / \mathrm{J}$ & $7,79 E+12$ \\
\hline Poscosecha & & & & & & $1,47 E+15$ \\
\hline $\begin{array}{l}\text { 15. Equipos } \\
\text { poscosecha }\end{array}$ & & $2,33 \mathrm{E}-03$ & kg/ha.año & $1,13 E+13$ & $\mathrm{seJ} / \mathrm{kg}$ & $2,64 \mathrm{E}+10$ \\
\hline $\begin{array}{l}\text { 16. Energía eléctrica } \\
\text { secado }\end{array}$ & & $8,76 E+08$ & $\mathrm{~J} / \mathrm{t}$ & $2,77 E+05$ & $\mathrm{seJ} / \mathrm{J}$ & $2,43 E+14$ \\
\hline $\begin{array}{l}\text { 17. Infraestructura } \\
\text { planta secado }\end{array}$ & & $9,11 E+02$ & $S / . / t$ & $1,35 E+12$ & $\mathrm{seJ} / \mathrm{sol}$ & $1,23 E+15$ \\
\hline Servicios & & & & & & $4,73 E+14$ \\
\hline $\begin{array}{l}\text { 18. Mano de obra } \\
\text { temporal }\end{array}$ & 38 & $1,69 E+08$ & J/ha.año & $2,80 E+06$ & $\mathrm{seJ} / \mathrm{J}$ & $4,73 E+14$ \\
\hline
\end{tabular}


Tabla № 2: Flujos emergéticos del sistema de producción de café orgánico.

\begin{tabular}{|c|c|c|c|c|c|}
\hline Símbolo & Flujo & Ecuaciones & Valor obtenido & Unidades & $\%$ \\
\hline I & Total recursos naturaleza & $I=R+N$ & $3,84 \mathrm{E}+15$ & seJ/ha.a & 56,01 \\
\hline $\mathbf{R}$ & $\begin{array}{l}\text { Recursos renovables de } \\
\text { la naturaleza }\end{array}$ & $R=R 1+R 2+\ldots+R i$ & $2,35 E+15$ & seJ/ha.a & 34,28 \\
\hline $\mathrm{N}$ & $\begin{array}{l}\text { Recursos no renovables } \\
\text { de la naturaleza }\end{array}$ & $\mathrm{N}=\mathrm{N} 1+\mathrm{N} 2+\ldots+\mathrm{Ni}$ & $1,49 E+15$ & seJ/ha.a & 21,73 \\
\hline $\mathrm{F}$ & Total recursos economía & $F=M+S n$ & $3,01 E+15$ & seJ/ha.a & 43,99 \\
\hline$M$ & $\begin{array}{l}\text { Materiales de la } \\
\text { economía }\end{array}$ & $M=M 1+M 2+\ldots+M i$ & $2,72 E+15$ & seJ/ha.a & 39,71 \\
\hline $\mathrm{Sr}$ & $\begin{array}{l}\text { Servicios renovables de } \\
\text { la economía }\end{array}$ & $\begin{array}{c}\mathrm{Sr}= \\
(0.38)^{*}(\text { mano_obra })\end{array}$ & $1,80 E+14$ & seJ/ha.a & 2,62 \\
\hline Sn & $\begin{array}{l}\text { Servicios no renovables } \\
\text { de la economía }\end{array}$ & $\begin{array}{c}\mathrm{Sn}= \\
(0.62)^{*}(\text { mano_obra })\end{array}$ & $2,93 E+14$ & seJ/ha.a & 4,28 \\
\hline$Y$ & Emergía utilizada & $Y=I+F$ & $6,85 E+15$ & seJ/ha.a & 100,00 \\
\hline
\end{tabular}

doble contabilidad. Los servicios renovables de la economía corresponden al 38\% de los servicios, esto significa que el $38 \%$ de la mano de obra temporal es renovable (5). Dentro de los recursos no renovables de la naturaleza solo se ha considerado el flujo de la pérdida de suelo.

Para el grupo de materiales de la economía se ha considerado 13 flujos emergéticos de los 18 que tiene el sistema. Estos 13 flujos son considerados como recursos no renovables de la economía.

Dentro de los servicios no renovables de la economía se ha considerado el $62 \%$ de la mano de obra temporal (5).

Finalmente, se constituyeron en dos grupos: total recursos de la naturaleza y total recursos de la economía, para después calcular los índices emergéticos.

En la Tabla 3 se presentan los índices emergéticos que han sido calculados para el sistema de producción de café orgánico. Los índices calculados fueron seis: transformidad, que consistió en estimar el total de flujos incorporados al sistema $(Y)$ entre el valor de la energía del producto logrado (Ep). Este valor ha sido calculado tomando como base la cantidad de J/ha.año del café, que es de 1.01E+10. También se estimó el valor de la transformidad tomando como base el valor total de producción de las 12075 has, el cual asciende a un total de 1918946 kilogramos de café pergamino producido por cada hectárea y

Tabla № 3: Índices emergéticos del sistema de producción de café orgánico.

\begin{tabular}{lccc}
\hline \multicolumn{1}{c}{ Índices } & Ecuaciones & Valor & Unidad \\
\hline Tr = Y/Ep (energía del producto) & $\mathrm{Tr}=\mathrm{Y} / \mathrm{Ep}$ & $4,75 \mathrm{E}+07$ & $\mathrm{seJ} / \mathrm{J}$ \\
Renovabilidad (\%R) & $\mathrm{Ren}=(100)^{*}(\mathrm{R} / \mathrm{Y})$ & 49,78 & $\%$ \\
Tasa de eficiencia emergética (EYR) & $\mathrm{EYR}=\mathrm{Y} / \mathrm{F}$ & 2,00 & Adimensional \\
Tasa de carga ambiental (ELR) & $\mathrm{ELR}=(\mathrm{N}+\mathrm{F}) /(\mathrm{R})$ & 1,01 & Adimensional \\
Tasa de inversión emergética (EIR) & $\mathrm{EIR}=\mathrm{F} / \mathrm{I}$ & 1,00 & Adimensional \\
Índice de sostenibilidad emergética $(\mathrm{SI})$ & $\mathrm{ESI}=\mathrm{EYR} / \mathrm{ELR}$ & 1,99 & Adimensional \\
\hline
\end{tabular}


en un año de producción.

El indicador de renovabilidad emergética - sostenibilidad fue estimado dividiendo el valor de los recursos renovables incorporados sobre el total de flujos. Este indicador es expresado en porcentaje.

La tasa de eficiencia emergética (EYR) fue estimada a partir de la relación entre el total de flujos incorporados (Y) y la aportación de los actores económicosociales $(F)$, y presenta un valor de 2,27 . La tasa de carga ambiental (ELR) fue calculada sumando los recursos no renovables incorporados por la naturaleza y el total de aportaciones de la economía entre el total de recursos renovables incorporados por la naturaleza. La tasa de inversión emergética (EIR) fue estimada luego de relacionar la aportación de la economía y los recursos de la naturaleza. Finalmente, el índice de sostenibilidad emergética (SI) fue calculado al dividir la tasa de eficiencia emergética entre la tasa de carga ambiental.

\section{DISCUSIÓN}

Los resultados finales de la aplicación de la metodología emergética planteada por Odum (1), muestra una transformidad de 6,79E+05 seJ/J. La transformidad del sistema es obtenido a través de la sumatoria de los valores de todos los flujos de emergía que compone el producto final (café pergamino). Este resultado es dividido entre la energía del producto generado por el sistema. Esta es una medida de la eficiencia del sistema estudiado. Cuanto mayor sea su valor, menor es la eficiencia del sistema.

Sarcinelli y Ortega (6) reportan valores de $1,05 \mathrm{E}+10 \mathrm{seJ} / \mathrm{J}$ en el café orgánico del Brasil. En consecuencia, el valor de transformidad del café orgánico de la región Junín es mucho más eficiente que el café orgánico de Brasil. De acuerdo con la relación de flujos incorporados al sistema $(Y)$, existe una mayor eficiencia de generación de energía en el producto final al salir del sistema de producción de café orgánico. Esto lógicamente se debe a que en el proceso existe una menor incorporación de recursos provenientes de la economía $(3,01 \mathrm{E}+15 \mathrm{seJ} / \mathrm{ha}$.a $)$. Las inversiones que realizan los pequeños productores de café son marcadamente inferiores a las que realizan los productores de café orgánico de Brasil. Los recursos de parte de la economía que incorporan los productores de café de la región Junín representan el 43,99\% respecto al $87,5 \%$ reportado en el LEIA (7). Además, la producción de café orgánico en ambos sistemas difiere en magnitud o economía de escala.

Sobre la transformidad que toma como base la masa seca de café orgánico obtenido del sistema, Ortega (8) presenta un valor de $3,45 \mathrm{E}+13 \mathrm{sej} / \mathrm{kg}$ para el café tradicional, muy superior al encontrado en el presente estudio, 3,57E $+12 \mathrm{sej} / \mathrm{Kg}$. Esto confirma que el sistema de producción de café orgánico en el Perú, por tratarse de un sistema que preconiza la utilización de recursos naturales renovables, es más eficiente que el café tradicional de Brasil.

La renovabilidad (\%R) o la porción de recursos renovables utilizados en el sistema de producción de café orgánico, es una medida directa de la sostenibilidad. Cuanto más alto sea su valor, mayores son las oportunidades del sistema para mantenerse en el largo plazo. El sistema orgánico presenta una renovabilidad de $34,28 \%$. Este resultado es superior comparado con el que reporta Giannetti y otros (9), 28\% para la etapa de producción agrícola de café de Nicaragua. La razón de que el café peruano presenta mayor renovabilidad se debe principalmente a que los valores de los recursos renovables son mucho mayores respecto al total de 34,28\%. Dentro de este resultado está incluido el $38 \%$ de renovabilidad de parte del total aportado por los servicios, es decir, de los servicios renovables. El mayor valor de aporte de la economía es debido al flujo de parte de los materiales, 39,71\%. En este último resultado, al igual que en los recursos renovables, también se ha incluido el $62 \%$ 
restante de la aportación de los servicios, para este caso considerado como servicio no renovable. Fernandez (5) manifiesta que para los productos convencionales provenientes de la agricultura como el café, maíz, soja y frutas los valores de renovabilidad van desde 5 a 10\%.

La tasa o razón de rendimiento emergético (EYR), definida como la emergía total utilizada por el sistema (Y) dividida por la emergía de los insumos de la economía $(F)$, es una medida de ganancia en energía primaria disponible para el uso de la sociedad. Los sistemas utilizan en sus procesos recursos naturales y recursos provenientes de la economía, estos últimos considerados como recursos naturales que fueron previamente obtenidos por la naturaleza, procesados por otros sistemas y puestos a disposición para el uso de la sociedad. En este caso la EYR es igual a 2,27 . Valores superiores a 1 indican que el sistema fue capaz de disponer energía primaria para la sociedad y cuanto mayor es este valor, mayor es la eficiencia del sistema en la utilización de los recursos invertidos de la economía (10). En el presente estudio el valor de la tasa de eficiencia energética fue de 2,27, valor superior a 1 y que muestra mayor nivel de eficiencia emergética.

La tasa de carga ambiental (ELR), es una medida del impacto ambiental derivado del sistema productivo. En teoría, si no ocurren inversiones por el hombre, los flujos emergéticos renovables disponibles localmente deberían sostener un ecosistema maduro sujeto a las restricciones impuestas por el medio ambiente, en este caso ELR sería igual a cero. Cuando ocurren inversiones de flujos emergéticos externos al sistema, el patrón de desarrollo es diferente del ecosistema original. De esta forma, ELR mide la distancia entre el sistema en estudio y el ecosistema original y puede ser interpretado como el estrés ocasionado al medio ambiente por el sistema productivo de café orgánico. Cuanto más alto es su valor, mayor la distancia del sistema original, y mayor será el impacto, o el estrés en el ecosistema asociado. En el presente estudio el valor hallado de ELR fue de 1,92, elevado en relación con el valor reportado por Sarcinelli y Ortega (6), 0,11. De modo que el impacto al medio ambiente, donde se produce el café orgánico, es mayor en Junín que en Brasil.

La tasa de inversión emergética (EIR) es la razón entre los recursos de la economía y de la naturaleza e indica la inversión de la sociedad para producir un bien, en relación con la contribución de la naturaleza. Puede ser utilizado para evaluar la eficiencia o la competitividad del sistema productivo en la utilización de recursos invertidos de la economía. Mientras menor sea el valor, será más competitivo. En el presente estudio ese valor es de 0,79, el cual es inferior al reportado por Giannetti y otros (9), 2.40 para el café de Brasil, y al mismo tiempo inferior al reportado por Waureck y otros (11), 3,70 para el trigo ecológico. Por lo tanto, el sistema de producción de café en Satipo es más competitivo que los sistemas de café y trigo ecológicos mencionados.

El índice de sostenibilidad emergética (SI), es una medida de la contribución potencial del sistema (EYR) por unidad de carga ambiental, o impacto ambiental (ELR), impuesto al área ocupada por el sistema de producción de café. En teoría, el menor valor posible del índice de sostenibilidad emergética es cero, cuando ELR es infinito. Pero el mayor valor del índice de sostenibilidad emergética, infinito, ocurre cuando EYR tiende al infinito, situación que solamente ocurre para ecosistemas maduros no explotados ( $F$ igual a cero). Por tanto, cuanto más alto el valor de dicho indicador, menor impacto por unidad de energía primaria disponible para la sociedad. Según Brown y Ulgiati (12), valores de $\mathrm{SI}$ inferiores a 1 indican sistemas que consumen recursos, en tanto que valores superiores a 1 indican sistemas que contribuyen con la aportación de recursos para uso de la economía sin afectar el equilibrio del medio ambiente. Así, los valores inferiores a 1 están asociados a las economías altamente desarrolladas y orientadas para el consumo.

En el presente estudio se obtuvo un valor de 
1,19. De acuerdo con Brown y Ulgiati (12), este valor indicaría un menor consumo de recursos, lo que es aceptado en la presente investigación dado que existe una proporción menor de recursos aportados por la economía, que es de $43,99 \%$. Con respecto al aporte de la naturaleza, presenta un valor superior de $56,01 \%$. También afirman que valores superiores a diez están referidos a economías poco desarrolladas industrialmente. Esta afirmación no se ajusta a los resultados alcanzados debido a que el valor logrado para el presente indicador ambiental no corresponde a la clasificación de economías desarrolladas.

En conclusión, es posible identificar los elementos del sistema debido al buen nivel de organización. El sistema presenta un mayor aporte de recursos de la naturaleza con respecto al aporte de la economía. Los indicadores emergéticos demuestran que el sistema de café orgánico presenta mayor eficiencia, mayor renovabilidad, menor impacto al ecosistema y buena contrapartida de la naturaleza a las inversiones respecto a lo que los productores invierten. Por lo tanto, el sistema de producción de café orgánico de la región Junín es sostenible ambientalmente.

\section{Agradecimientos}

A la Universidad Continental por el financiamiento de este trabajo de investigación, asimismo a los productores de la Cooperativa Agraria Cafetalera de Satipo Ltda.

\section{REFERENCIAS BIBLIOGRÁFICAS}

1. Odum HT. Environmental accounting. Emergy and environmental decision making. New York: John Wiley \& Sons; 1996.

2. Siche R. Avaliacao ecológicatermodinámica e económica de nacoes: o Perú como estudo de caso [Tesis Doctorado en Ingeniería de los Alimentos]. Campinas, Brasil: Universidade Estadual de Campinas; 2007.

3. Lomas P, Di Donato M, Ulgiati S. La Síntesis Emergética: una valoración de los servicios de los ecosistemas con base termodinámica. Revista Ecosistemas [Internet]. 2007; [fecha de consulta 28 de noviembre de 2010]. 16 (3):[pp. 36-44]. Disponible en: http://www.revistaecosistemas. net/articulo.asp? Id $=497 \& I d$ Categoria $=1$ \&tipo = portada

4. Guillén HA. Contabilidad ambiental usando emergía: Caso 2: Sostenibilidad de sistemas agro-silvícolas y agroindustriales en Chiapas, México. [Internet]. México: Universidad Autónoma de Chiapas [fecha de consulta: 6 de noviembre de 2011]. Disponible en: http://www.bvsde. paho.org/bvsaidis/mexico 13/092.pdf

5. Fernandez C. Avaliacao da sustentabilidade ampliada de produtos agroindustriais [Tesis Doctorado en Ingeniería de los Alimentos]. Campinas: Universidad Estadual de Campinas; 2008.

6. Sarcinelli O, Ortega E, editores. Emergy analysis and bookkeeping accounting of conventional and organic coffee production in Brazil. Editado por Ulgiati $\mathrm{S}$, Ortega E. Proceeedings of IV Biennial International Workshop "Advances in Energy Studies"; Campinas, Laboratory of Ecological Engineering, Food Engineering School. Junio 16, 2004.

7. LEIA-Laboratorio de Engenharia Ecológica-Unicamp. Brasil. FEA Unicamp. 2006. [Actualizado al 19 de setiembre del 2012; fecha de acceso 
22 de enero de 2013]. Disponible en: http://www.unicamp.br/fea/ortega/

8. Ortega $E$, Zanghetin M, Takahashi F. Conceitos básicos sobre a biosfera, os ecossistemas e a economia humana. Módulo 1. Como funciona a natureza. Sao Paulo: Laboratorio de Engenharia Ecológica da Unicamp, 2008. pp. 34.

9. Giannetti BF, Ogura $Y$, Bonilla SH, Almeida CM. Accounting emergy flows to determine the best production model of a coffe plantation. Energy Policy [Internet]. 2011 [fecha de consulta: 16 de setiembre de 2011 ]; 39: [pp. 73997407]. Disponible en: http://www. advancesincleanerproduction.net/ papers/journals/2011/2011_Energy_ Policy_\%20Coffee.pdf

10. Ulgiati S. Modeling matter, energy and information flows through ecological and economic systems, within a "zero emission" framework. 4th European Conference on Ecological Modelling [CD-ROM]. Bled, Slovenia; 2004.

11. Waureck A, Eurich J, Weirich Neto PH, Rocha $\mathrm{CH}$. Estudo da sustentabilidade a través de índices emergéticos em dois sistemas de producao de trigo. Seminario Internacional "Experiencias de agendas 21: os desafios do nosso tempo". Fortaleza, Universidade Estadual de Ponta Grossa. 2009. 14.

12. Brown $M$, Ulgiati, S. Energy quality, emergy, and transformity: H.T. Odum's contribution to quantifyng and understanding systems. Ecodinamica [Internet]. 2004 [fecha de consulta: 12 de agosto del 2012]; 5: [58-67 pp.]. Disponible en: http:// www.onb.it/writable/editoriale/ Brown_Ulgiati_5_2004_Energy\%20 quality, $\% 20$ Emérgy $\% \overline{2} 0$ and $\% 20$ Transformity.pdf 